\title{
Pluralismus von Recht und Ordnung ${ }^{1}$
}

\author{
Franz von Benda-Beckmann
}

\begin{abstract}
Der Beitrag befasst sich mit der Frage, wie sich die Beziehungen zwischen Recht und Ordnung in rechtspluralistischen Kontexten darstellen. Die wechselhafte Geschichte der Minangkabau in West Sumatra, bei denen staatliches, islamisches und traditionelles Adatrecht koexistieren, zeigt, dass die unterschiedlichen Inhalte der Teilrechtsordnungen und die mit ihnen verbundenen Autoritäten zwar nicht notwendigerweise zu sozialen Konflikten führen müssen, die Ordnung jedoch riskanter machen. Das Potential für soziale Konflikte nimmt zu, wenn sich inhaltlich unterschiedliche rechtliche Entwürfe der sozialen Organisation auf unterschiedliche Legitimationsgrundlagen berufen können, welche die legitime Macht der hinter einer Ordnung stehenden Autoritäten relativieren.
\end{abstract}

Keywords: Rechtspluralismus; Kolonialstaat

\section{Einleitung}

Der Begriff Rechtspluralismus weist auf die Möglichkeit, dass es innerhalb derselben sozialen Formation eine Mehrzahl von rechtlichen Ordnungsvorstellungen geben kann. Im Folgenden möchte ich auf einige Fragen eingehen, welche eine derartige plurale Rechtsordnung aufwirft, wenn man über Ordnung nachdenkt. Ändert Rechtspluralismus unser Verständnis des Verhältnisses? Macht Rechtspluralismus Ordnung einfacher oder schwieriger oder gar riskanter? Rechtspluralismus scheint die beiden wichtigsten tragenden Säulen des Rechts- und Ordnungsdenkens zu unterminieren: die Idee der gemeinsamen Werte und Normen, die, in Durkheim'scher Tradition, oft als die wichtigste Bedingung von Ordnung betrachtet werden; und/oder die Rolle der die Ordnung tragenden und wiederherstellenden Autoritäten, des Souveräns in seinen mannigfaltigen Erscheinungsformen, wie sie eher durch Hobbes und Weber entwickelt wurden.

In den jüngeren Globalisierungsdebatten hat das Interesse für Rechtspluralismus und seine Folgen für die Macht des Staates, Ordnungen zu gestalten und durchzusetzen, zugenommen. Jedoch sind Rechtspluralismus und damit verbundene Probleme von Recht und Ordnung keineswegs etwas Neues, wie uns ein Blick auf unsere eigene Geschichte, vor allem aber auch auf die Geschichte und Gegenwart ehemaliger kolonialer Staaten deutlich zeigt. Das Beispiel, auf das ich im Folgenden zurückgreifen werde, entstammt der Geschichte der Minangkabau in West Sumatra in Indonesien, wo meine Frau und ich lang-

1 Leicht überarbeite Fassung des Vortrags, den der Verfasser am 3. Mai 2007 im Rahmen der Vorlesungsreihe Riskante Ordnungen in Leipzig gehalten hat. 
jährige Feldforschungen gemacht haben. ${ }^{2}$ Hier gab es einen Rechtspluralismus schon lange bevor diese Gesellschaften in den kolonialen Staat inkorporiert wurden. Dieser entstand mit der Islamisierung, die neue politische, wirtschaftliche, soziale und religiöse Ordnungsmuster und Autoritäten einbrachte, die fortan mit dem Minangkabauschen Adat und seinen Autoritäten koexistierten. Adat ist eine umfassende Kategorie, die das kulturelle, symbolische und rechtliche Universum der Minangkabau andeutet. Vor fast 200 Jahren kam mit dem kolonialen Staat eine neue Ordnungsmacht hinzu, womit der Rechtspluralismus seine in Indonesien typische triangulare Konstellation von Adat, Islam, Staat bekam. Diese blieb, wenn auch in unterschiedlicher Form und Gewichtung auch nach Indonesiens Unabhängigkeit im Jahre 1945 erhalten. Im Laufe der letzten zehn Jahre hat der Einfluss neuer transnationaler Einflüsse zugenommen, durch welche sowohl rechtlich-politische Modelle wie Good Governance, Demokratie etc. durch externe Akteure verbreitet werden, als auch orthodoxe Versionen des Islam und des islamischen Rechts. So entstand im Laufe der Geschichte ein breites Repertoire von Ordnungsvorstellungen, in dem ältere oft durch neuere überlagert wurden, aber selten ganz verschwanden. Es handelt sich dabei um historisch wandelbare, dynamische Konstellationen von Ordnungsvorstellungen und die sie verwaltenden Autoritäten.

\section{Rechtspluralismus und Ordnung}

Bevor ich näher auf diese Entwicklungen eingehe, sollen erst einmal einige allgemeine Vorbemerkungen zu Recht, Rechtspluralismus und Ordnung folgen. Recht beinhaltet Ordnungsvorstellungen, normative Blaupausen für das gesellschaftliche Zusammenleben im weitesten Sinne: für die Organisation des Staates, der Wirtschaft, der unterschiedlichen sozialen Verbände und Organisationsformen; aber auch Grenzziehungen für erlaubtes und verbotenes Verhalten; Verfahrensvorschriften, die regeln, was passieren soll, wenn rechtliche Ordnungsmuster nicht befolgt werden, wenn Konflikte entstehen, und wer die Ordnung wiederherstellen darf oder soll. Rechtspluralismus bezieht sich auf die Möglichkeit, dass es innerhalb derselben sozialen Formation zur selben Zeit eine Mehrzahl von rechtlichen Ordnungsvorstellungen gibt (von Benda-Beckmann 2006b). Weiter kann Rechtspluralismus auch die unterschiedlich legitimierte Autorität von Personen oder Instanzen umfassen, die zur Interpretation und Wahrung von Recht und Ordnung ermächtigt sind. Damit ist die Bindung von Recht an den Staat aufgebrochen und die Möglichkeit eröffnet, die staatliche Rechtsordnung und die ihr zu Grunde liegende Legitimation nur als eine unter mehreren, gleichzeitig existierenden zu erfassen. Rechtspluralismus ist keine Theorie und keine empirisch feste Größe. Er ist ein sensibilisierender Begriff, der auf die Möglichkeit einer derartig komplexen Konstellation aufmerksam macht und auf die Relativität und Variabilität der empirischen Erscheinungsformen hinweist. Die gleichzeitige Existenz von Rechtsordnungen kann sich auf den gesamtgesellschaftlichen Bereich oder nur auf bestimmte Teilbereiche wie Familienverhältnisse, Vererbung oder die politische Organisation beziehen. Sie kann unterschiedliche Formen und Dynamiken

2 Seit 1999 untersuchen wir die Folgen der Dezentralisierungspolitik in West Sumatra. Unsere frühere Forschung Mitte der 1970er Jahre erlaubt uns eine gute Übersicht über die Veränderungsprozesse, siehe Benda-Beckmann 2006a. 
annehmen. Es kann sich um ein weitgehendes Nebeneinander und/oder Ineinander und/oder Gegeneinander der einzelnen Rechtsformen handeln.

Die mich interessierende Frage ist, wie sich Rechtspluralismus zur Ordnung verhält. Ordnung hat unterschiedliche Bedeutungen, die wiederum weitgehend davon abhängen, was man als Gegensatz zur Ordnung ansieht. Wenn ich im Folgenden über Ordnung spreche, habe ich meist drei Gegensätze im Kopf. Einmal Ordnung als das Maß, das gesellschaftliches Handeln durch Regeln, Prinzipien, Institutionen und Verfahren ordnet im Gegensatz zu Freiheit und Autonomie. Ordnung wird hier verstanden als Insel im Meer der Unbestimmtheit und Offenheit. Zum anderen geht es um Ordnung im Sinne von geordnet sein - im Gegensatz zur Unordnung. Der Gegensatz zwischen Ordnung und Unordnung ist hier negativ konnotiert. Damit sind Chaos, Konflikte, Gewalt und Orientierungslosigkeit verbunden. Drittens kann das Gegenteil von Ordnung eine andere Ordnung sein. In all diesen Fällen sind Ordnung und ihr Gegensatz immer relativ. Von extremen Situationen abgesehen entsteht eine Vorstellung von Ordnung weitgehend im Auge des Betrachters. Was für den einen Freiheit oder eine sinnvolle Ordnung ist, ist für den Anderen Unordnung oder eine schlechte Ordnung.

Ordnung ist ein umfassenderer Begriff als Recht und die darin enthaltenen Ordnungsvorstellungen. Rechtliche Ordnungsvorstellungen sind nur eine Art der Betrachtung, eine große Brille oder Meßlatte, mit der man Ordnung bzw. ihr Gegenteil bestimmen kann. Gemessen daran, kann die empirische Ordnung als Unordnung wahrgenommen werden. Aber neben rechtlichen Ordnungsvorstellungen gibt es auch die gelebte Ordnung, d.h. Ordnung als Zustandsbeschreibung, ausgedrückt in erfolgreich und friedlich institutionalisierten Verhaltensabläufen, einschließlich der Prozesse, die die Ordnung wiederherstellen. Rechtspluralismus kompliziert dieses Verhältnis. Das Anpassen an die Ordnungsvorstellungen des einen Rechts, zum Beispiel des Staates, erweist sich zugleich als ein Abweichen von den Regeln des anderen, zum Beispiel des Adatrechts oder der Sharia und umgekehrt. Was unordentlich und ungeordnet aus der Sicht der Rechtsordnungen ist, ist jedoch nicht unbedingt ungeordnet im Sinne der gelebten Ordnung. In der gelebten Ordnung müssen die Gegensätze zwischen den unterschiedlichen Rechtsordnungen nicht unbedingt zu entsprechenden sozialen Konflikten führen. Man kann sich streiten, aber man kann sich auch arrangieren, und man kann das eine tun und das andere nicht lassen. Ordnung sollte deshalb nicht darauf reduziert werden, inwiefern sie normativen Ordnungsvorstellungen entspricht.

\section{Ordnung und Recht im vorkolonialen West Sumatra}

Die Minangkabau in West Sumatra waren in Nagari, weitgehend autonomen Dorfrepubliken, organisiert. Es gab keine die Nagari übergreifende Ordnung. „Dorfordnung“ war also Ordnung per se. Die ursprüngliche Logik des Adat war es, dass es sich bei den Nagari um einen freiwilligen Zusammenschluss von im Prinzip unabhängigen und gleichwertigen in Matriklans vereinigten Abstammungsgruppen handelte. Die Zugehörigkeit zu diesen wichtigsten sozialen, wirtschaftlichen und politischen Einheiten der Dorfgesellschaft wurde über die weibliche Linie bestimmt. Die Nachfolge in politische Ämter und die Vererbung von Gütern verlief ebenfalls über die weibliche Linie. Innerhalb der Familie dominierte der Muttersbruder als Autoritätsperson. Der Vater gehörte zu einer anderen Abstammungsgruppe als seine eigenen Kinder und übte dort, als Muttersbruder, Autorität aus. Alle Klan-internen Angelegenheiten wurden im Prinzip innerhalb der Klans entschieden. Der Adat-Dorfrat des Nagari, der aus den Oberhäuptern der 
ältesten Abstammungsgruppen bestand, war in Angelegenheiten zuständig, die das ganze Dorf betrafen. Je nach Anzahl der großen Abstammungsgruppen konnten das 30 bis 60 Oberhäupter sein. Der Dorfrat funktionierte auch als Berufungsinstanz in Angelegenheiten, die innerhalb der Gruppe nicht friedlich und einverständlich beigelegt werden konnten.

Auf dieser Grundlage beruhte das Prinzip, dass richtige und gültige Entscheidungen nur einvernehmlich getroffen werden konnten. Ein Prozess gemeinsamen Beratschlagens musste zur konsensualen Entscheidung führen. Entscheidungsfindung auf der Basis von politischer Gleichheit war also eine der verfassungsrechtlichen Grundlagen. Neben diesem egalitären gab es auch ein hierarchisches Prinzip, das an der segmentären Struktur der Klans orientiert war. Probleme und Konflikte mussten zunächst auf der untersten Ebene unter der Leitung des Gruppenoberhaupts gelöst werden. Wenn das nicht glückte, ging man eine Stufe weiter bis zur Ebene des ganzen Klans, und wenn auch dort keine Entscheidung getroffen werden konnte, zum Adat-Dorfrat. Diese Ideen formen das Grundverständnis von Adat-Demokratie und legitimer Entscheidungsfindung.

\section{Islam und Adat-Gewohnheitsrecht}

Die erste große Herausforderung dieser Ordnung kam mit dem Islam, der sich gegen Ende des 16. Jahrhunderts in West Sumatra ausbreitete. Die islamischen Prinzipien von Herrschaft, Familienorganisation und Vererbung widersprachen deutlich den matrilinearen Strukturen des Minangkabauschen Adat. Zunächst wurde der Islam nur als Religion angenommen. Im weltlichen Bereich mussten sich islamische Prinzipien und auch Autoritäten dem Adat unterordnen. Zu Beginn des 19. Jahrhunderts kam es dann zu einer „Zurück zur Sharia"-Bewegung. Diese sogenannten Padri versuchten, erst mit friedlichen Mitteln und später in einem blutigen Bürger- bzw. Religionskrieg, ihre in ihren Augen ungläubige Gesellschaft zu reformieren. Es ging um den Verfall der Sitten, um Hahnenkämpfe, Opium-Rauchen, Glücksspiel und um unzüchtige Kleidung der Frauen. Man versuchte auch, die Nagari-Verfassung auf ein theokratisches Modell umzustellen. Auf Ansuchen einiger Adat-Führer intervenierten die Holländer, die bisher nur einen Handelsposten an der Küste gehabt hatten, in diesem Krieg, schlugen die Padri und verleibten das Minangkabausche Hochland ihrem Kolonialreich ein. In der Zwischenzeit hatten Adat und Islam zwar Frieden geschlossen, aber es war zu spät, um die politische Unabhängigkeit der Nagari zurückzugewinnen. In dieser Zeit bildete sich ein neues ideologisches Grundprinzip heraus, das den unverbrüchlichen Zusammenhalt von Adat und Islam zum zentralen Merkmal Minangkabauscher Kultur und Identität machte. „Adat beruht auf der Sharia, die Sharia auf Adat". Die gegensätzlichen Ordnungen von Adat und Islam und ihre Führer hatten sich zu politischen und ideologischen Kompromissen bereit gefunden; jedenfalls auf einem sehr allgemeinen und von konkreten Problemen abstrahierenden Niveau. Probleme blieben, wenn es in konkreten Lebenslagen um den Vorrang der beiden Ordnungsmuster ging. Wen musste man fragen, wenn man heiraten wollte? An wen wendete man sich, wenn man einen Streit geschlichtet haben wollte? Und vor allem und immer der heißeste Punkt: Wer waren die Erben eines Mannes? In bäuerlichen Gesellschaften wie den Minangkabau ist die Vererbung von großer Wichtigkeit, weil sie den hauptsächlichen Zugang zu Land und damit zum wichtigsten Produktionsmittel darstellt. Die Spannung zwischen der Dominanz der weiblichen Linie, der Vererbung des Vermögens an die Schwesterskinder, nicht an die eigenen Kinder des Mannes, und dem Wunsch von Vätern, Vermögen an die eigenen Kinder zu übertragen, sieht man in allen matrili- 
nearen Gesellschaften. Bei den Minangkabau war die Spannung zusätzlich religiös beladen, denn der Islam und das islamische Recht hatten natürlich eine ganz andere Vorstellung bezüglich der Verteilung von Gütern nach dem Tode. Diese beruhte auch auf ganz anderen sozial-ökonomischen Ordnungsvorstellungen, nämlich der Teilbarkeit und Verhandelbarkeit von Vermögen - im Gegensatz zur Minangkabauschen Gesellschaft und ihrem System von kontinuierlich vererbten Vermögenskomplexen der Abstammungsgruppen, das auf Mehrung des wirtschaftlichen Vermögens der Abstammungsgruppen und seine Bewahrung für die kommenden Generationen ausgerichtet war. Die Verfügungsfreiheit der Lebenden war deshalb eingeschränkt. Der Gruppe sollte kein zukünftiges Vermögen durch Verfügungen unter Lebenden entzogen werden.

\section{Rechtspluralismus im kolonialen Staat}

Eine neue Konstellation von Rechtspluralismus und eine neue tatsächliche Ordnung begann, als die Holländer West Sumatra und seine Nagari in den kolonialen Staat inkorporierten. Zwar hatten die Holländer den Minangkabau in ihren ersten Verträgen zugesichert, sie würden sich nicht in die lokalen Angelegenheiten einmischen, aber wie das so üblich war, hielt man sich nicht an die Verträge. Die koloniale Regierung gebrauchte die Nagari als Basiseinheiten für die Organisation ihrer indirekten Herrschaft. Dabei unterstützten die Holländer die Adat-Strukturen bewusst, um ein Gegengewicht zum politisch viel virulenteren Islam zu haben. Die vorkoloniale Adat-Organisation wurde jedoch in mancher Hinsicht geändert. Um einen hauptsächlichen Ansprechpartner zu haben, setzte man eine Art Adat-Bürgermeister ein, denn mit 60 gleichberechtigten Oberhäuptern per Dorf zu verhandeln, war ihnen zu umständlich. 1914 bekamen die Nagari eine GemeindeVerfassung, die auch einen Dorfrat installierte.

Seitdem hatten alle Nagari bis in die heutige Zeit eine mehr oder weniger ausgeprägte dualistische politische Organisation, die auf unterschiedlichen Legitimationen beruhte. Auf der einen Seite die staatlich geregelte Dorfverwaltung, bei der neben dem Dorfbürgermeister unterschiedliche Formen von Dorfräten die Bevölkerung repräsentieren sollten. In diesen waren die „Drei Säulen“ vertreten: Adat-Älteste, die religiösen Führer und die „Intellektuellen“. ${ }^{3}$ In den Räten spielten Adat-Älteste immer eine wichtige Rolle, ihnen gehörten jedoch nie alle nach Adat Berechtigten an. Parallel dazu war jedoch die Fortsetzung einer auf jeden Fall ideologisch „reinen“ Adat-Struktur vorgesehen. Deren höchstes Organ war der Adat-Dorfrat, der im Prinzip alle nach Adat berechtigten Oberhäupter umfasste. Diese Dorfräte waren über lange Perioden durch die Kolonialregierung und nach der Unabhängigkeit durch den Indonesischen Staat nicht offiziell anerkannt, funktionierten aber auch ohne Anerkennung mehr oder weniger effektiv und wurden durch die koloniale Verwaltung geduldet und durchaus geschätzt.

\section{Dezentralisierung und Reorganisation der Dorfverwaltung}

Auch nach der Unabhängigkeit Indonesiens im Jahr 1945 baute die unterste staatliche Verwaltungsstruktur auf den Nagari auf. 1983 kam es dann zu einem neuen Bruch. Das

3 Die Intellektuellen (cerdik pandai) waren wegen ihrer Bildung geschätzte Personen, die kein Adat- oder religiöses Amt bekleideten. 
Modell der Desa-Dörfer wurde aus Java importiert. Desa waren kleinere administrative Einheiten, die viel stärker als die Nagari in eine hierarchische Befehlsstruktur eingebettet waren. Diese Reorganisation der Dorfverwaltung führte in West Sumatra dazu, dass alle Nagari in mehrere Desa aufgeteilt wurden und damit die räumliche Kongruenz zwischen staatlichen und traditionellen Verwaltungseinheiten aufgehoben wurde. Adat-Autoritäten hatten in der Desa-Verwaltung keine offizielle Rolle mehr.

Nach dem Ende der neuen Ordnung mit dem Fall Suhartos im Jahre 1998 begann wieder eine neue Phase, die Zeit der Reformen, die orde reformasi. Diese wird unter anderem durch einen viel größeren politischen Freiraum gekennzeichnet, in dem eine Politik der Dezentralisierung verfolgt wird. Dies war die Folge des Nachgebens der Zentralregierung gegenüber dem zunehmenden Protest der Regionen über den schiefen Finanzausgleich und die fehlende lokale Autonomie. Zugleich wurde dieser Prozess durch internationale und ausländische Geberorganisationen, dem IMF und der Weltbank, der Asian Development Bank, UNDP, USAID, AUSAID und auch der GTZ, vorangetrieben und unterstützt.

In der Provinz West Sumatra hat man diese neue Freiheit genutzt, um die Dorfverwaltung neu zu organisieren. Man kehrt seit 2000 zum Nagari als unterster Verwaltungseinheit zurück. Neben einer demokratisch organisierten Dorfverwaltung mit gewähltem Bürgermeister und Dorfparlament wird auch wieder ein Adat-Dorfrat errichtet bzw. anerkannt, der sich für Adat einsetzen, Streitfälle in Adat-Angelegenheiten schlichten und mit der Dorfverwaltung die Dorfressourcen verwalten soll. Dieses „Zurück zum Nagari“ wird als Zurück zum Adat interpretiert, und hierdurch hat sich, sicher in den ersten Jahren nach der Reform, die Balance zwischen Adat, Islam und Staat zugunsten des Adat geändert. Am auffallendsten ist dies im symbolischen und ideologischen Bereich. Adat ist wieder „,in“. Für die regionalen Politiker dient die Berufung auf ihr Adat dazu, um gegenüber der Zentralregierung die Eigenständigkeit Minangkabaus zu betonen. Die Provinzverordnung und die Distriktsverordnungen sind voller Adat-Rhetorik. Adat ist die Zauberformel, mit der alle sozialen Übel aus der Welt geschafft werden sollen: Die Einheit und der Zusammenhalt innerhalb der Nagari soll verstärkt werden, die Jungen werden die Älteren wieder respektieren, die Adat-Führer die Nagari-Resourcen nicht mehr an externe Investoren verscherbeln. Diese Rhetorik und die häufig damit verbundene romantische Verklärung der Adat-Vergangenheit hört man vor allem von Mitgliedern der älteren Generation. Sie wird vor allem durch die alten und neuen Eliten getragen. Die jüngere Generation, die nicht mehr erlebt hat, wie das Leben im Nagari war, ist sehr viel skeptischer. Aber Adat hat wieder Prestige und man sieht überall, wie lange Zeit unbesetzte Ämter von AdatAutoritäten wieder neu besetzt werden. Dieser „Minangkabausche Weg“ hat auch außerhalb West-Sumatras viel Aufmerksamkeit auf sich gezogen, sowohl bei Vertretern anderer ethnischer oder regionaler Organisationen in Indonesien als auch bei DonorOrganisationen. Er wird als ein wichtiger Ansatz betrachtet, in dem lokale traditionelle demokratische Strukturen und Führer wieder eine größere Rolle in einem Prozess bekommen, der zu mehr Demokratie und einer weitergehenden Einbeziehung der Bürger führen soll. Good governance avant la lettre.

Diese Entwicklung beeinflusste auch die islamische Seite des Dreiecks. Die Rückkehr zum Nagari betraf in erster Linie Adat und seine Revitalisierung im Bereich der dorfpolitischen Organisation und des Bodenrechts. Seitens islamischer Kreise wurde neben der „Zurück zum Nagari“- eine „Zurück zur Surau“-Bewegung initiiert. Surau waren Gemeinschaftshäuser, in denen Kinder und Jugendliche in Adat und Islam unterwiesen wurden. Mit dem „Zurück zur Surau“ wurde die Bedeutung der Religion in der neuen Ordnung im Nagari betont; die Tatsache, dass neben Adat auch die religiösen Werte von Bedeutung waren; dass Adat und Islam eine unverbrüchliche Einheit formten. Während 
die frühere Formulierung der Beziehungen zwischen Adat und Islam, „Adat beruht auf der Sharia, die Sharia auf Adat", auf eine prinzipielle Gleichwertigkeit deutete, wird in jüngerer Zeit die Ausdrucksweise „Adat beruht auf der Sharia, die Sharia auf dem Koran" bevorzugt, die eine leichte Überlegenheit des religiösen Rechts angibt. In den häufigen Diskussionen über die Bedeutung dieses Verhältnisses - in jedem Jahr gibt es in Minangkabau ein oder zwei Konferenzen zu dem Thema - wird der Kampf um die relative Bedeutung und erhoffte Überlegenheit eines der beiden Elemente in der Minangkabauschen Identität und einer wünschenswerten Ordnung geführt.

Dabei richtete sich der religiöse Druck nicht so sehr auf Fragen der politischen und wirtschaftlichen Organisation, sondern auf Normen und Werte im Alltagsleben. Es wird primär, etwas salopp gesagt, die Moralkeule geschwungen, und dies mit immer größerem Erfolg. Besonders, wie damals vor 200 Jahren, ist es ein Kampf gegen die sogenannten „gesellschaftlichen Krankheiten“ wie Drogen, Prostitution, Glückspiel, Korruption und für Frömmigkeit und islamische züchtige Kleidung der Frauen etc. Der Staat, meint man, habe bei der Bekämpfung dieser Übel versagt. Und weil der altmodische Adat diese Aufgabe allein nicht zu leisten vermag, ist nun auch der Islam gefragt. Neben der Revitalisierung des Adat sieht man also auch eine Revitalisierung des Islam in der öffentlichen Ordnung. Dies scheint zugleich auch eine zunehmende Islamisierung der Menschen zur Folge zu haben, und zwar relativ unabhängig von der Funktion, die sie ausüben, d.h. egal, ob sie Verwaltungsbeamte, Adat-Älteste oder Dorfbürgermeister sind. So sieht man, dass fortschrittlich-demokratische Reformer der Distriktsverwaltung Verordnungen erlassen, wonach heiratswillige Personen Korankenntnisse nachweisen müssen und die weiblichen Verwaltungsbeamten auf jeden Fall freitags islamisch gekleidet sein sollen. Ähnliche Verordnungen werden durch Dorfregierungen erlassen, Dorfregierungen, deren Bürgermeister zuvor der Vorsitzende des Adat-Dorfrats war. Was als neue Bedeutung von Adat begann, scheint in eine Richtung zu gehen, die zu einer viel größeren Bedeutung des Islam in der Staat-Islam-Adat-Einheit führt.

\section{Rechtspluralismus als riskante Ordnung}

Was können wir nun daraus lernen? Sind pluralistische Rechtsordnungen riskante Ordnungen, riskanter als andere? Um meine Ansicht kurz zusammenzufassen: Ich meine ja. Zwar ist das nicht notwendigerweise der Fall, aber die Wahrscheinlichkeit ist eher größer.

Natürlich kann man sich eine pluralistische Ordnung auch als geordnet vorstellen. Das war auch, was koloniale Herrscher und ihre offiziellen Rechtsordnungen versuchten. Sie wollten diesen Pluralismus über eine begrenzte Anerkennung von ethnisch oder territorial verankerten Gewohnheits- oder religiösen Rechten so ordnen, dass die Vielfalt zu einem geregelten Nebeneinander würde; einem Nebeneinander bzw. Untereinander von Parallelwelten, wie man heute vielleicht sagen würde. Dies war allerdings nur eine rechtliche Konstruktion, eine aus der Sicht des Staates. Auch die anderen Rechtsordnungen konnten ihrerseits mehr oder weniger deutliche Regeln darüber haben, wie mit den anderen Ordnungen umzugehen sei, in welchem Maße im islamischen Recht zum Beispiel Gewohnheitsrecht, Adat und Urf in der islamischen Rechtsterminologie, oder staatliches Recht als geltend anerkannt werden konnte. In den kolonialen Gebieten hatte man also, auf rechtlichem Niveau, einen Pluralismus von Rechtspluralismus-Konstruktionen.

Rechtliche Konstruktionen der Gültigkeit der jeweiligen Rechts- und Ordnungssphären mit ihrer Verteilung von politischer, sozialer und wirtschaftlicher Ordnungsmacht 
bedeuten nicht unbedingt Unordnung oder Konflikt. Aber das Potential dafür ist groß. Mit dem Pluralismus von Ordnungsvorstellungen hat jede einzelne ihre Selbstverständlichkeit verloren. Man braucht sich Alternativen nicht neu auszudenken - sie sind schon da, sind theoretisch verfügbar und werden einem praktisch vorgelebt. Das Potential für Konflikte nimmt zu, wenn sich die unterschiedlichen Teilordnungen auch auf unterschiedliche Legitimationsgrundlagen berufen, d.h. wenn zum Beispiel neben der und gegen die staatliche Verfassung auf göttliches, heiliges Recht oder vorstaatliche Traditionen zurückgegriffen wird. Für die die Selbstverständlichkeit einer jeden Ordnungsvorstellung sowieso schon relativierenden inhaltlichen Alternativen wird mit der Berufung auf das Recht eine zusätzliche Legitimationsgrundlage geliefert, die auch die legitime Macht der hinter den jeweiligen anderen Ordnungen stehenden Autoritäten relativiert. Auch geringfügige Probleme werden dann leicht zum Anlass genommen, um allgemeine weltanschauliche oder religiöse Auseinandersetzungen rechtlich auszutragen. Hier wird der Rechtspluralismus außerhalb seiner staatlich-rechtlichen Regelung wirklich irritierend für diejenigen, die das Recht der Ordnung und zum Ordnen aus einer unumstößlichen Quelle ableiten. Distanz zur dominanten rechtlichen Ordnungsideologie wird schnell als ein gegen diese gerichteter politischer Akt interpretiert. In den Augen vieler staatstreuer Juristen und Rechtstheoretiker ist diese Idee des Rechtspluralismus dann auch bestenfalls Unsinn. Sie werden die Meinung Hobbes teilen, der solche Denker zu den „kleinen Würmern“ zählte, „einer Krankheit des Commonwealth, die politische Weisheit vorspiegelnd sich die Freiheit nehmen, die absolute Macht in Frage zu stellen und so mit den fundamentalen Gesetzen des Commonwealth zu spielen.“4

Der Blick in die Minangkabausche Geschichte hat gezeigt, dass man fast alle denkbaren Formen und Prozesse von Koexistenz wahrnehmen kann. Teils wurden diese Gegensätze in kriegerischen Auseinandersetzungen ausgetragen, wie im Padri-Krieg, der Unterwerfung Minangkabaus durch die Holländer und der Befreiung Minangkabaus von der holländischen Kolonialherrschaft. Die inhaltlichen Gegensätze der unterschiedlichen Ordnungen führten aber nicht zwangsläufig zu entsprechenden sozialen Konflikten. Sie konnten in einem routinierten Spannungsverhältnis aufgefangen werden, in dem es immer nur um kleine Verbesserungen der eigenen Position in der Balance im Verhältnis Adat und Islam, Islam und Staat, Adat und Staat ging. Die Gegensätze wurden auf umfassendem Niveau über lange Perioden als gut integriert definiert - Adat und Islam als Harmonie und als unverbrüchliche Essenz des Minangkabau-Seins; Adat, Staat und Islam als identitätsstiftende Dreieinheit, die drei miteinander verwobenen Fäden, wie die Minangkabau sagen, welche die Identität des Minangkabauschen Staatsbürgers ausmachen. Warum sollte man wählen, wenn man auch kombinieren konnte? Warum bei einer EheschlieBung nicht nur Adat, sondern auch religiöse und staatlich-rechtliche Vorschriften beachten und die seltenen Fälle, in denen man das nicht wollte, zu Ausnahmen deklarieren? Dasselbe gilt für Personen. Warum sollte man nicht gleichzeitig Adat-Oberhaupt, religiöse Autorität und moderner Staatsbeamter oder Politiker sein? Im Laufe der Zeit konnte es somit zu hybriden Mischformen kommen. Der durch den Staat geregelte Adat-Rat, so wie

4 Dies ist meine Übersetzung von ,the little wormes, ... a desease of a commonwealth, pretenders to political prudence who, by taking the liberty of disputing against absolute power, are meddling with the fundamental lawes to the molestation of the commonwealth" (Hobbes 1968, 375). 
er jetzt in der Neuordnung der Dorfverwaltung erscheint, ist so eine hybride Institution, die staatliche und Adat-Regelung und Legitimität miteinander verschmilzt.

Aber die schlafenden Hunde, das Risikopotential pluralistischer Ordnungen, sind nie verschwunden; auch wenn sie nicht bellen, träumen sie oft. Denn man kann alles ja auch ganz anders sehen. Den Adat-Rat kann man als geglückte Integration von staatlichen und Adat-Prinzipien betrachten. Man kann ihn, wie das im heutigen Dezentralisierungsdiskurs noch überwiegend der Fall ist, als Ikone für die Wiederauflebung des Adat betrachten. Er kann aber genau so gut für einen durch den Staat pervertierten Adat stehen, dem man die reine Adat-Struktur gegenüberstellt. Was wie Adat und Islam Jahre miteinander verschmolzen war, teils ideologisch, teils institutionell, kann wieder entzerrt werden, entzweit im wahrsten Sinne des Wortes.

Externe Einflüsse und Bindungen mit auswärtigen Organisationen oder Netzwerken sind dabei ein Faktor, der das Konfliktpotential vergrößert. Bei der Entstehung und den Transformationen pluralistischer Rechtskonstellationen spielen Außeneinflüsse eine wichtige Rolle. Natürlich kann sich Pluralismus auch innerhalb der Gesellschaft ohne diese herausbilden. Außeneinflüsse vereinfachen und beschleunigen allerdings das Entstehen von pluralistischen Ordnungen, wenn Personen und Organisationen in die Gesellschaft eintreten und mit friedlichen oder gewalttätigen Mitteln neue Ordnungsvorstellungen propagieren. Im Zeitalter der Globalisierung sind alternative Konzepte vermehrt auch über die neuen Medien verfügbar, ohne dass man lange nach ihnen suchen müsste.

Das sieht man in allen Phasen der Minangkabauschen Geschichte: bei der Einführung des Islam durch Händler und Gelehrte, bei der Ankunft der Holländer, bei der Erlangung der Unabhängigkeit und auch bei den heutigen Demokratisierungs- und Islamisierungswellen. Es waren keinesfalls immer von außen eindringende Einflüsse. Es waren oft auch Ideen, die die Minangkabau selbst herbeigeholt hatten. Das war der Fall bei den drei berühmten Minangkabauschen Pilgern, die 1803 in Mekka mit dem Wahabismus in Berührung kamen und die Padri Bewegung gründeten. Es war auch der Fall in den ersten Jahrzehnten des 20. Jahrhunderts, als indonesische Intellektuelle - darunter viele Minangkabau - westeuropäische Demokratievorstellungen aus Holland und moderne islamische Demokratievorstellungen aus der Türkei holten. Diese neuen Modelle entsprachen nicht der zutiefst undemokratischen rechtlichen und tatsächlichen Ordnung kolonialer Staaten. Wenn Mitglieder der Gesellschaft eine demokratische Ordnung verlangten, wurden sie sehr schnell zu Staatsfeinden deklariert, gefangen genommen oder verbannt.

Außeneinflüsse werden im Laufe der Zeit zu Bestandteilen der internen Ordnung und sind dann vielleicht gar nicht mehr, oder nur noch für den Historiker, als ehemaliger AuBeneinfluss erkennbar. Ich meine, und dies ist mein letzter Punkt, dass es ein besonders wichtiger Aspekt dieser riskanten Konstellationen ist, inwieweit die jeweiligen Teilordnungen weiterhin in externe Organisationen oder Netzwerke ein- oder angebunden bleiben. Je mehr das der Fall ist, desto größer ist die Wahrscheinlichkeit, dass man sich nicht, oder weniger leicht, arrangiert; dass man nicht eher Ruhe gibt, bis man selbst die dominante oder universale Ordnung geworden ist. Auch in der heutigen Zeit ist dies der Fall sowohl bei der Demokratisierung als auch der Islamisierung, die beide in enger Vernetzung mit ausländischen Organisationen und vor allem auch Geldgebern vorangetrieben werden. Was Jahre miteinander verbunden oder verschmolzen war, wie Adat und Islam, teils ideologisch, teils institutionell, kann wieder entzerrt werden und in seiner reinen Form, in der es vielleicht nie existiert hatte, konzeptualisiert und als neue bzw. uralte Ordnungsvorstellung propagiert werden. Was aus seiner internen Integration gelöst oder gerissen wird, wird oft in eine umfassendere translokale Gemeinschaft oder in ein Netz- 
werk integriert. Diese Verbindungen und die mit ihnen verbundenen Ressourcen werden dann im Kampf um die Vorherrschaft der eigenen Ordnung oder gar deren Universalisierung im Rahmen der pluralistischen Konstellation eingesetzt. Im heutigen Minangkabau ist der Zusammenhalt zwischen Adat, Islam und Staat noch so stark, dass sich diese Spannungen nicht, oder noch nicht, in neuen gewalttätigen Auseinandersetzungen manifestiert haben. Aber es bleibt weiterhin eine riskante Ordnung.

\section{Bibliographie}

Benda-Beckmann, F. and K. von (2006a) Changing one is changing all: Dynamics in the Adat-Islam-State triangle. In: Benda-Beckmann, F. and K. von (eds.) Dynamics of plural legal orders. Special Issue of the Journal of Legal Pluralism, 53/54: 239-270.

Benda-Beckmann, F. and K. von (2006b) The dynamics of legal pluralism. In: BendaBeckmann, F. and K. von (eds.) Dynamics of plural legal orders. Special Issue of the Journal of Legal Pluralism, 53/54: 1-41.

Hobbes, T. (1968[1651]) Leviathan. Ed. by C. B. Macpherson, Harmondsworth: Pelican Books.

Franz von Benda-Beckmann leitet zusammen mit Keebet von Benda-Beckmann seit Sommer 2000 die Projektgruppe „Rechtspluralismus“ am Max-Planck-Institut für ethnologische Forschung in Halle; von 1981-2000 Professor für Recht und Entwicklung (Universität Wageningen/NL); 1970 Promotion (Jura) zu Rechtspluralismus in Malawi; 1979 Habilitation im Fach Ethnologie an der Universität Zürich, wo er von 1972-1978 als Assistent am Ethnologischen Seminar arbeitete; 1974-1975 Feldforschung in West Sumatra zu vermögens- und erbrechtlichen Prozessen; Mitte der 1980er Jahre Forschungen auf Ambon in den Molukken zu sozialer Sicherung und Ressourcenrechten der dörflichen Bevölkerung; seit 1999 Untersuchungen zu den Auswirkungen der Dezentralisierungspolitik in West Sumatra; weitere Forschungsinteressen: methodologische und theoretische Fragen der Rechtsethnologie; seit 2002 Honorarprofessor für Ethnologie (Universität Leipzig); seit 2004 Honorarprofessor für Rechtspluralismus (Universität HalleWittenberg).

E-mail: fbenda@eth.mpg.de 